\title{
Discovery of a recombination dominant plasma: a relic of a giant flare of Sgr A*?
}

\author{
S. Nakashima ${ }^{1}$, M. Nobukawa ${ }^{1}$, H. Uchida ${ }^{1}$, T. Tanaka ${ }^{1}$, \\ T. G. Tsuru ${ }^{1}$, K. Koyama ${ }^{1,2}$, H. Uchiyama ${ }^{3}$ and H. Murakami ${ }^{4}$ \\ ${ }^{1}$ Department of Physics, Graduate School of Science, Kyoto University, \\ Kitashirakawa Oiwake-cho, Sakyo-ku, Kyoto 606-8502, Japan \\ email: shinya@cr.scphys.kyoto-u.ac.jp \\ ${ }^{2}$ Department of Earth and Space Science, Graduate School of Science, Osaka University, \\ 1-1 Machikaneyama, Toyonaka, Osaka 560-0043 \\ ${ }^{3}$ Science Education, Faculty of Education, Shizuoka University, \\ 836 Ohya, Suruga-ku, Shizuoka 422-8529, Japan \\ ${ }^{4}$ Department of Information Science, Faculty of Liberal Arts, Tohoku Gakuin University \\ 2-1-1 Tenjinzawa, Izumi-ku, Sendai, Miyagi 981-3193, Japan
}

\begin{abstract}
We present the discovery of out-flow like plasma emissions with the Suzaku and ASCA data. Those plasmas have a size of $\sim 150$ pc. Remarkably, the southern plasma is in a recombination dominant phase, which is not predicted by standard shock heating. A plausible scenario is either photoionization due to strong jet-like X-rays from $\mathrm{Sgr} \mathrm{A}^{*}$ or rapid cooling due to adiabatic expansion of a blowout plasma from the Galactic center about $10^{5}$ years ago.
\end{abstract}

Keywords. Galaxy: center - X-rays: ISM - ISM: jets and outflows

\section{Introduction}

One of the most remarkable Galactic center (GC) activities is past giant flares of the central supermassive black hole, Sagittarius A* (Sgr A*). X-ray observations found timevariable Fe I K $\alpha$ emission from dense molecular clouds, best interpreted as X-ray echoes of giant Sgr A* flares a few hundred years ago (Inui et al. 2009; Ponti et al. 2010). GeV $\gamma$-ray observations revealed a large scale bipolar emission, the so-called Fermi bubbles, suggesting a jet-like activity about a million years ago ( $\mathrm{Su}$ et al. 2010). If such past Sgr A* activities are common events, another relic of a Sgr A* flare should be present in the vicinity of the GC. Based on this idea, we investigate the X-ray data.

\section{Observations and analysis}

\subsection{X-ray images}

We used the GC survey data taken by ASCA (Tanaka et al. 1994) and Suzaku (Mitsuda et al. 2007). Figure 1 shows the X-ray images of the GC region. From the ASCA image (Figure 1a), we found large diffuse emission 1-2 degree extending from the GC to the north and south. It exhibits a bipolar outflow-like morphology. We refer to these emissions as GC North and GC South in this paper. Due to the better photon statistics of the Suzaku data, Figure 1b more clearly shows the excess of GC North and GC South, even though not all of this field is covered by the Suzaku observations. On the other hand, the Suzaku hard X-ray image shows no prominent excess to the north and south of the GC (Figure 1c). 
(a) ASCA 1.5-3.0 keV

(b) Suzaku 1.0-3.0 keV (c) Suzaku 5.0-8.0 keV
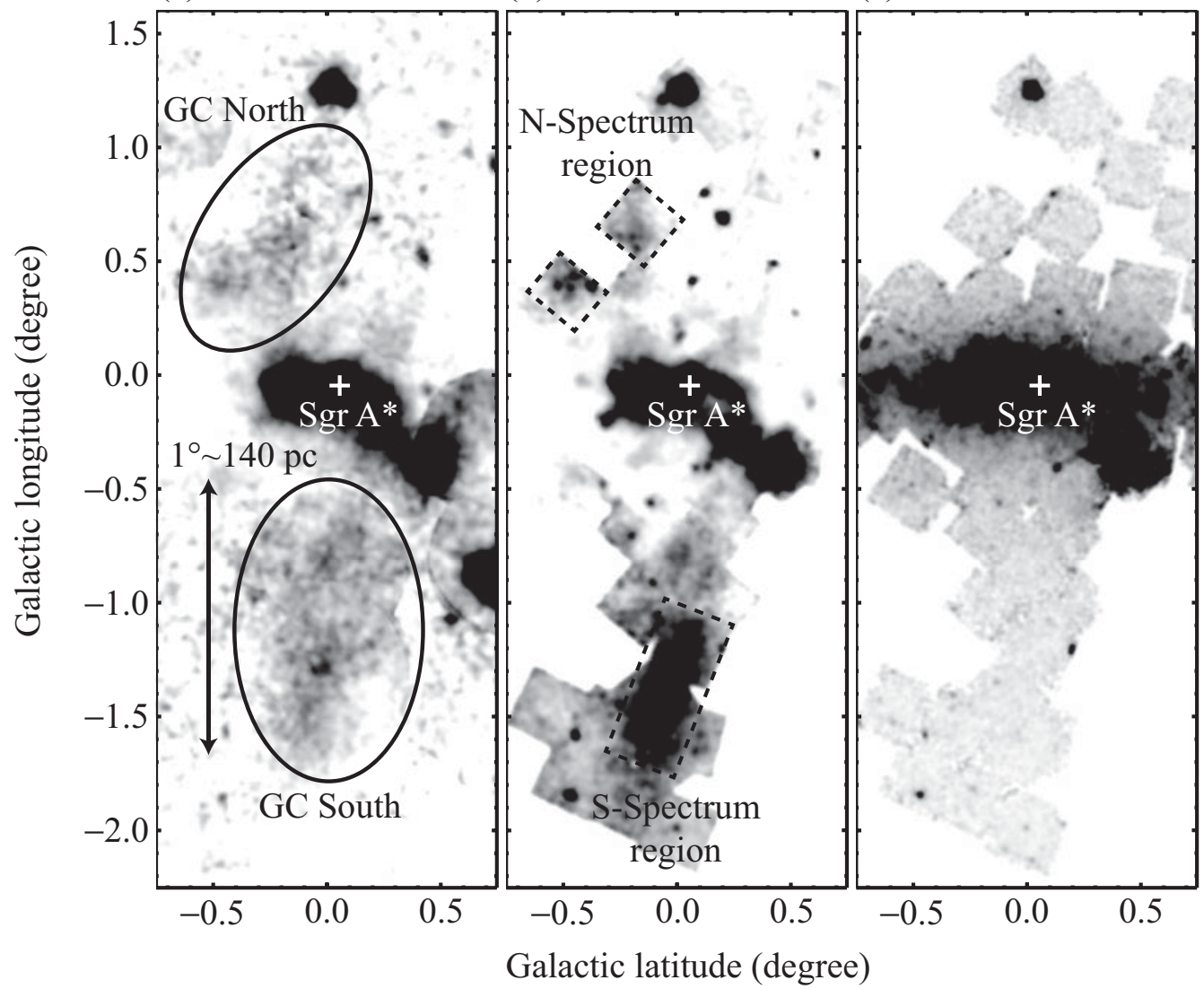

Figure 1. X-ray images of the GC in the (a) $1.5-3.0 \mathrm{keV}$ band with $A S C A$, (b) $1.0-3.0 \mathrm{keV}$ band with Suzaku, and (c) 5.0-8.0 keV band with Suzaku.

\subsection{GC South}

We analyzed a spectrum of the brightest part of GC South. Figure 2 shows the spectrum extracted from the rectangle region in Figure 1 (the S-Spectrum region). Prominent emission lines from highly ionized atoms ( $\mathrm{Ne} . \mathrm{Mg}, \mathrm{Si}, \mathrm{S}, \mathrm{Ar}$, and $\mathrm{Fe}$ ) and a neutral $\mathrm{Fe}$ line are detected. The emission in the 5-10 keV band including the Fe K-shell lines are most likely of Galactic background (BG) origin. We investigated the BG spectrum using nearby Suzaku observations and modeled it (see Nakashima et al. 2013 for details). Applying this BG model, the $5-10 \mathrm{keV}$ band spectrum of GC South is well represented (the dashed line in Figure 2), while the excess in the $0.7-5 \mathrm{keV}$ band still remains with emission lines.

We then added an optically-thin thermal plasma model in collisional ionization equilibrium (CIE). The electron temperature $\left(k T_{\mathrm{e}}\right)$, the emission measure $(E M)$, and abundances of elements (N, Ne, Mg, Si, S, and Fe) were allowed to vary. Since some Fe L-shell lines at $0.8 \mathrm{keV}$ and $1.2 \mathrm{keV}$ are not included in the model, we also modeled them with two Gaussians. Spectral fitting of this model gave a best-fit $k T_{\mathrm{e}}$ of $0.62 \mathrm{keV}$, but did not represent the data with $\chi^{2} / d o f=1378 / 350$ (Figure $2 \mathrm{~b}$ ). The residual at $2.0 \mathrm{keV}$ corresponds to $\mathrm{H}$-like $\mathrm{Si} \mathrm{K} \alpha$, and indicates that this CIE model underestimates the ionization state of Si. In addition, one can clearly see hump-like features around 2.5-3.0 keV and 


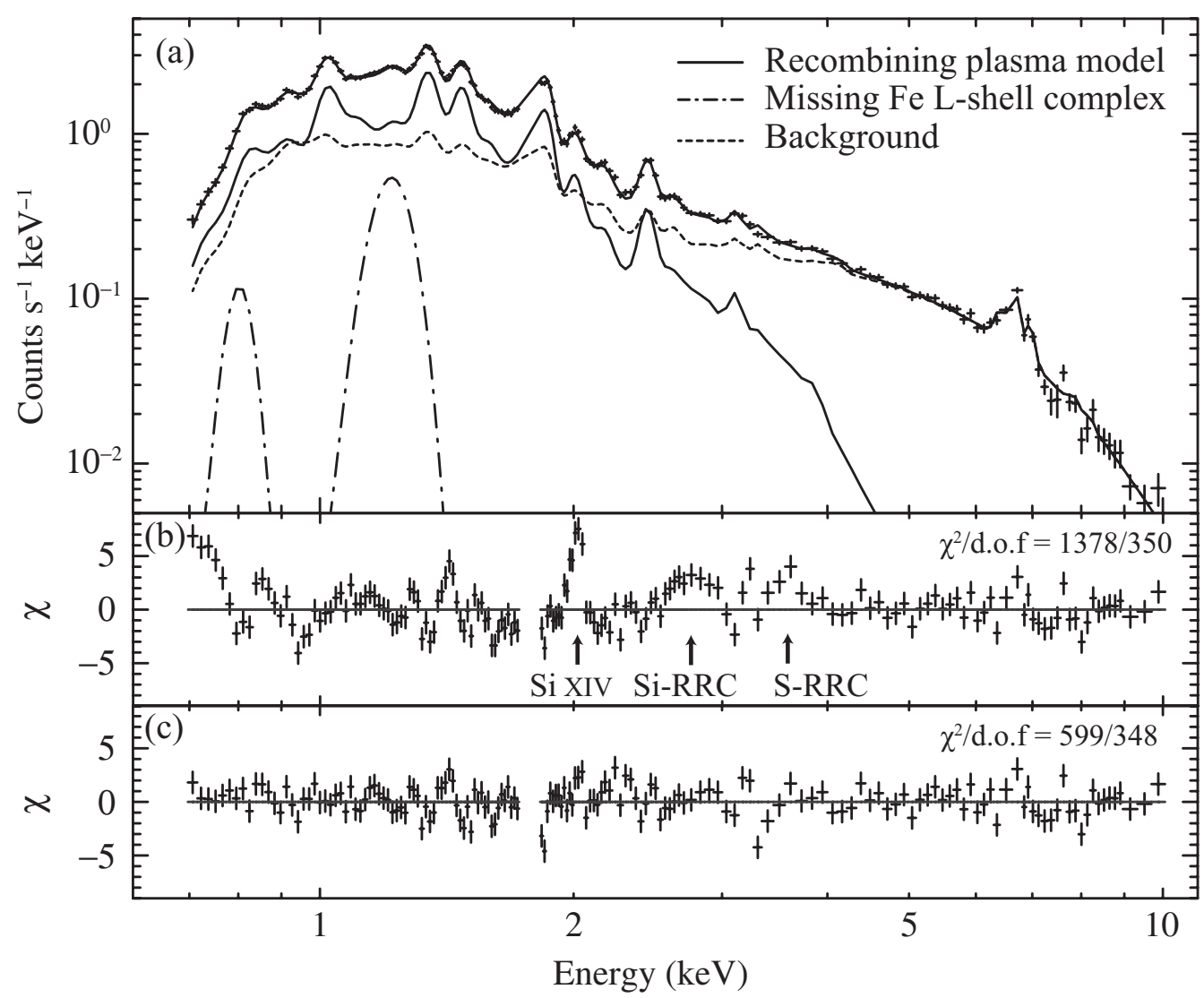

Figure 2. (a) Spectrum of GC South fitted with the CIE plasma and BG model. (b) Residuals between the data and the CIE plasma model. (c) Residuals between the data and the NEIJ model.

3.2-3.8 keV. These features are most likely due to the radiative recombination continua (RRCs) of Si and S, which are detected in some Galactic supernova remnants (SNRs; see Sawada \& Koyama 2012; Uchida et al. 2012 and references therein).

The RRC residuals strongly indicate that a recombining process dominates in the plasma. We thus applied a non-equilibrium ionization model (NEIJ in SPEX) that successfully reproduces the RRC features observed in some SNRs (Uchida et al. 2012; Yamauchi et al. 2013). The free parameters of this model is the initial ionization temperature $\left(k T_{\text {init }}\right)$, the electron temperature $\left(k T_{\mathrm{e}}\right)$, and the relaxation timescale of $n_{\mathrm{e}} t$, where $n_{\mathrm{e}}$ and $t$ are the number density of electrons and the elapsed time, respectively. The condition of $k T_{\text {init }}>k T_{\mathrm{e}}$ describes a recombining plasma (RP). Spectrum fitting of this model fairly improved the residuals, and gave a best-fit with $\chi^{2} / d o f=599 / 348$ (Figure 2c). The best-fit parameters are summarized in Table 1

\subsection{GC North}

We also analyzed a Suzaku spectrum of GC North, although the entire GC north region has not been observed yet. We extracted spectra from the dashed rectangle regions in Figure 1b, and merged them to increase the photon statistics. A background-subtracted spectrum is shown in Figure 3. Emission lines from highly ionized $\mathrm{Mg}, \mathrm{Si}$, and $\mathrm{S}$ are detected. 
Table 1. Best-fit parameters of GC South and GC North.

\begin{tabular}{llll}
\hline Parameter & Unit & GC South & GC North \\
\hline$N_{\mathrm{H}}$ & $\left(10^{22} \mathrm{~cm}^{-2}\right)$ & $0.70 \pm 0.03$ & $2.79 \pm 0.06$ \\
$k T_{\mathrm{e}}$ & $(\mathrm{keV})$ & $0.46 \pm 0.02$ & $0.81 \pm 0.02$ \\
$k T_{\text {init }}$ & $(\mathrm{keV})$ & $1.63_{-0.18}^{+0.27}$ & - \\
$n_{\mathrm{e}} t$ & $\left(10^{11} \mathrm{~s} \mathrm{~cm}^{-3}\right)$ & $5.3 \pm 0.5$ & - \\
$E M^{3}$ & $\left(10^{58} \mathrm{~cm}^{-3}\right)$ & $9.5 \pm 1.0$ & $4.3 \pm 0.3$ \\
$\mathrm{Ne}=\mathrm{O}$ & $($ solar $)$ & $0.39_{-0.04}^{+0.05}$ & 1 (fixed) \\
$\mathrm{Mg}$ & $($ solar) & $0.81 \pm 0.07$ & 1 (fixed) \\
$\mathrm{Si}$ & $($ solar) & $0.71 \pm 0.05$ & 1 (fixed) \\
$\mathrm{S}$ & $($ solar) & $0.79_{-0.08}^{+0.09}$ & 1 (fixed) \\
$\mathrm{Ar}=\mathrm{Ca}$ & $($ solar) & $1.9_{-0.5}^{+0.6}$ & 1 (fixed) \\
$\mathrm{Fe}=\mathrm{Ni}$ & (solar) & $0.10 \pm 0.02$ & 1 (fixed) \\
& & &
\end{tabular}

Notes:

${ }^{1}$ Fitted with the NEIJ model.

${ }^{2}$ Fitted with the CIE plasma model.

${ }^{3}$ Emission measure assuming a distance of $8 \mathrm{kpc}$.

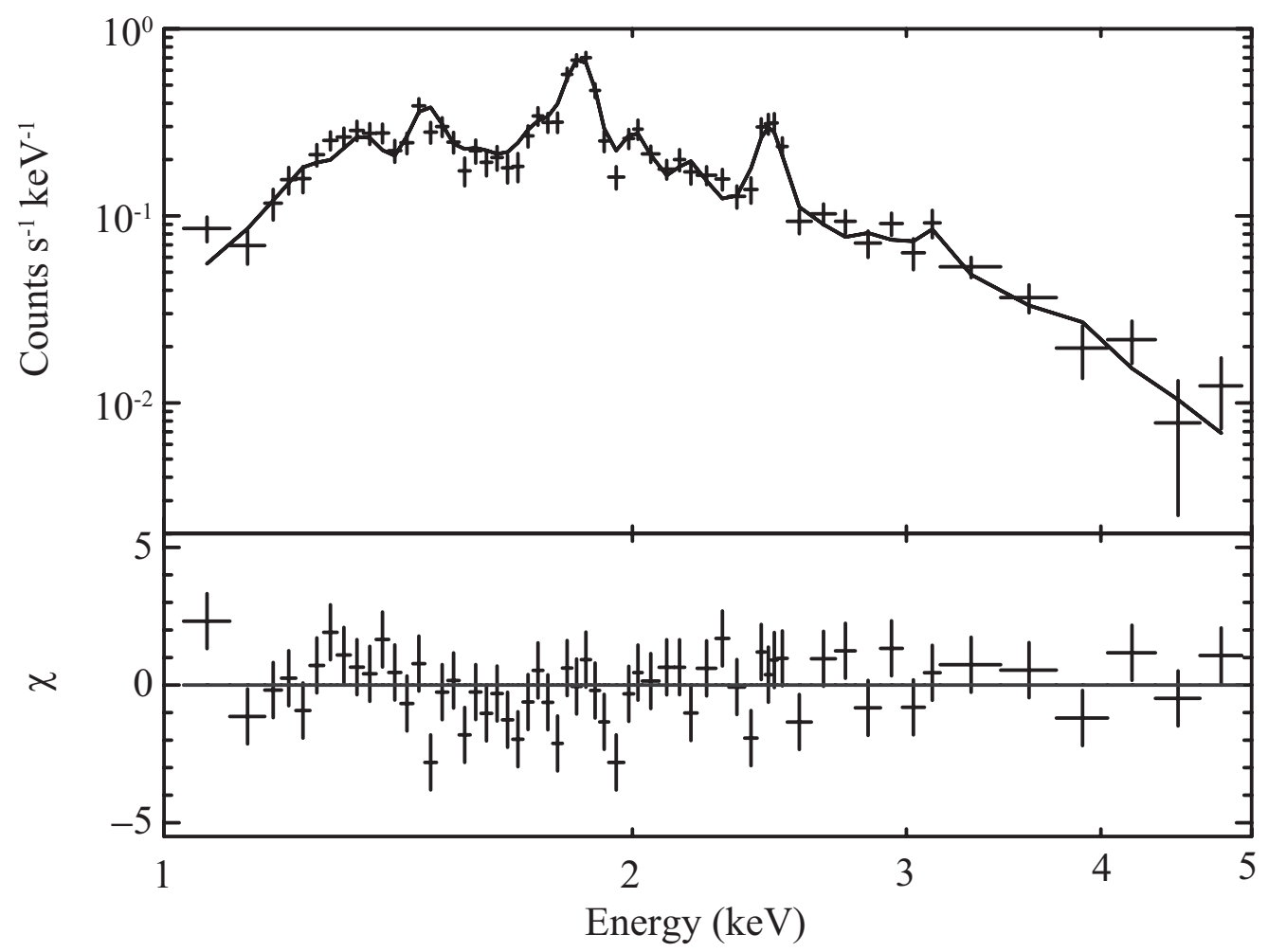

Figure 3. Spectrum of GC North fitted with the CIE plasma model.

We fitted the spectrum with the CIE plasma model, and obtained a reasonable fit with $\chi^{2} /$ dof $=156 / 117$. The parameters are listed in Table 1 . We also tried the RP model, but $n_{\mathrm{e}} t$ becomes larger than $10^{13} \mathrm{~s} \mathrm{~cm}^{-3}$, which indicates a CIE state. Therefore, a CIE state is favored, at least in the observed region.

\section{Discussion}

Absorption column densities of both GC North and GC South are consistent with those toward the GC, which were estimate from the near-infrared extinction (Gonzalez 
et al. 2012). We thus conclude that they are located at the GC distance of $\sim 8 \mathrm{kpc}$. The apparent size of GC North and GC South were then calculated to be $\sim 70 \mathrm{pc} \times 40 \mathrm{pc}$ and $\sim 100 \mathrm{pc} \times 40 \mathrm{pc}$, respectively. Assuming the length along the line of sight to be $40 \mathrm{pc}$, we estimated the volume of the plasmas and obtained a gas density of $0.1 \mathrm{~cm}^{-3}$ for both plasmas. Total thermal energy is $3 \times 10^{51}$ ergs and 10-100 times larger than that in a typical X-ray SNR. From the relaxation timescale of GC South, we estimate a plasma age of $\sim 10^{5}$ years.

The large thermal energy suggests that GC North and GC South are not SNRs but formed by past GC activity. One possibility is starburst activity, which causes blow-out of the plasma from the central star forming regions. Another is $\mathrm{Sgr} \mathrm{A}^{*}$ activity. If a jet had been emitted from Sgr A* and interacted with the dense interstellar medium, shockheated plasma would be formed. In any case, such activity would occur $\sim 10^{5}$ years ago.

The RP found in GC South is not predicted in the standard shock-heated plasma because the timescale of electron heating $(\sim 100$ years $)$ is much shorter than that of collisional ionization $\left(\sim 10^{4}\right.$ years $)$ in a low-density $\left(\sim 1 \mathrm{~cm}^{-3}\right)$ plasma. In order to form an RP, either a rapid decrease of the electron temperature or enhancement of ionization should occur. Blow-out of the plasma from the dense GC environment to the sparse Galactic bulge region would cause electron cooling due to rapid expansion of the plasma. Collimated X-ray radiation associated with Sgr A* jet could photo-ionize the plasma and also form the RP. Further investigation, especially GC North, is required to clarify the origin and the mechanism to form the GC North and GC South.

\section{References}

Gonzalez, O. A., Rejkuba, M., \& Zoccali, M., et al. 2012, A\& A 543, A13

Inui, T., Koyama, K., Matsumoto, H., \& Tsuru, T. G. 2009, PASJ 61, S241

Mitsuda, K., Bautz, M., \& Inoue, H., et al. 2007, PASJ 59, S1

Nakashima, S., Nobukawa, M., \& Uchida, H., et al. 2013, ApJ 773, 20

Ponti, G., Terrier, R., Goldwurm, A., Belanger, G., \& Trap, G. 2010, ApJ 714, 732

Sawada, M. \& Koyama, K. 2012, PASJ 64, 81

Su, M., Slatyer, T. R., \& Finkbeiner, D. P. 2010, ApJ 724, 1044

Tanaka, Y., Inoue, H., \& Holt, S. S. 1994, PASJ 46, L37

Uchida, H., Koyama, K., \& Yamaguchi, H., et al. 2012, PASJ 64, 141

Yamauchi, S., Nobukawa, M., Koyama, K., \& Yonemori, M. 2013, PASJ 65, 6 